\title{
Axial Wind-Borne Electrostatic Spray Research
}

\section{Z. T. Wang, J. L. Wen, X. Y. Wang, T. Q. Luo, X. N. Song}

School of Energy Resources and Power Engineering, Jiangsu University, Zhenjiang 212013, China

Email: wickol@ujs.edu.cn

\begin{abstract}
Axial wind-borne electrostatic spray is applied into practice. It could improve atomization and work efficiency. This paper analyzes the forces that act on the charged droplets, and the transport equations of charged droplets are established. The droplets movement velocity in the air flow and electric field could be obtained. Analyses these equations, we conclude that: 1) Droplets velocity is related to air velocityelectric field intensiontime and parameter $A_{1}=3 \pi \mu_{f} \mathrm{~d} f(R e) / m_{P}=18 \mu_{f} f(R e) / \rho_{P} \mathrm{~d}^{2}$, $A_{2}=q / m_{P}$, and would attenuate with the time going; 2) $A_{1}$ is inverse ratio to droplets diameter. When the droplets diameter decreasing, $v_{P}$ is faster close to $v_{f}$;3) The electric field force could make the droplets velocity bigger. When the electric field existing, the droplets follow characteristic is decreasing. On the base of theory analysis, the experimental research on axial wind-borne electrostatic spray is carried through. The charge characteristicdroplets size and the relations of atomization flow field and high voltage are obtained. The test results indicate: axial wind-borne electrostatic spray is superior to non-electrostatic spray. Finally, sterilization by electrostatic spray is carried out, the stcrilization efficiency is increased for $5 \% \sim 20 \%$.
\end{abstract}

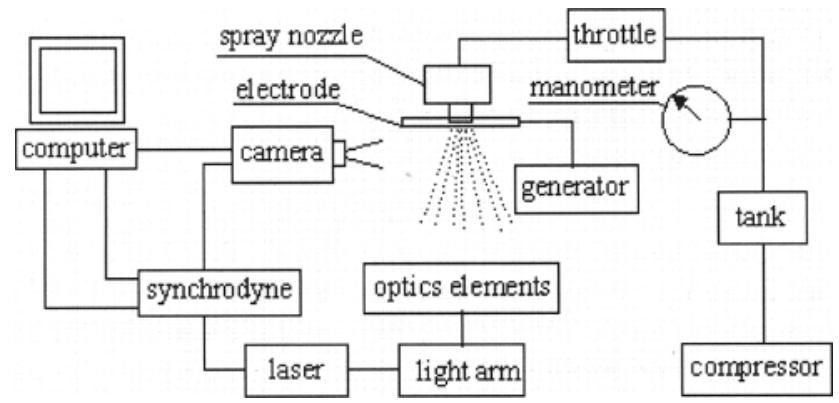

Figure: Electrostatic spray devices

\section{REFERENCES}

1. Law S E. Agricultural electrostatic spray application: a review of significant research development during the 20th century. Journal of Electrostatics, 2001; 51-52: 25-42

2. Wang Z T. The Research of Flue Gas Desulfurization by Electrostatic Spray. ZhenJiang: Jiangsu University, 2006

3. Wen J L, Tang Z H, Chen H L, Wang Z T. Electrostatic charged two phase flow equations. Transactions of the Chinese Society for Agricultural Machinery, 2006; 37(7): 59-61 